\title{
基于直接关系图法的碳氢燃料复杂化学机理简化
}

\author{
蒋勇邱 榕* \\ (中国科学技术大学火灾科学国家重点实验室, 合肥 230026)
}

\begin{abstract}
摘要: 采用直接关系图(DRG)法, 针对碳氢类氧化反应详细机理, 进行了不同指定精度水平的甲烷框架化学机 理简化研究. 通过 DRG 可以发现, 机理中存在强烈耦合的组分组, 在框架机理中, 这些组分组或同时保持或集体 去除. 研究表明, 给定阈值 $\varepsilon$ 为 0.01 的框架机理仅包含 182 个反应、涉及 27 种组分, 虽然给定阈值的精度并不高, 但所得框架机理和原详细机理关于火焰结构的计算结果吻合很好. 本研究发现, 开始组分仅给定燃料物质是 不够的, 当开始组分仅给定燃料物质, 则阈值 $\varepsilon$ 需很小, 形成的框架机理较大, 否则计算误差不可接受, 而开 始组分给定燃料和氧化剂(或 $\mathrm{N}_{2}$ ) 物质, 國值 $\varepsilon$ 可以取得较大, 形成的框架机理尺度可以较小, 而火焰模拟的精度 仍很高.
\end{abstract}

关键词：化学动力学简化；框架化学机理；直接关系图

中图分类号: 0643

\section{Reduction of Large Kinetic Mechanisms of Hydrocarbon Fuels with Directed Relation Graph}

\author{
JIANG Yong QIU Rong * \\ (State Key Laboratory of Fire Science, University of Science and Technology of China, Hefei 230026, P. R. China)
}

\begin{abstract}
The directed relation graph (DRG) method was used to reduce a detailed reaction mechanism to a skeletal mechanism for methane with specified accuracy. Strongly coupled species groups were observed in the reduction process, hence facilitating the identification of candidate skeletal mechanisms by eliminating or retaining the strongly coupled species in groups. The skeletal mechanism with an identified threshold value of 0.01 for methane oxidation and consisting of 27 species and 182 reactions was found to mimic the performance of the detailed mechanism with a high level of reliability. In this study, we found that the starting set could not simply consist of a single species such as the fuel otherwise the threshold value would need to be smaller and the created skeletal mechanism would have been larger. It simulates the flame structure well using the created skeletal mechanism in which the starting set of species consists of the fuel and oxidizer (or $\mathrm{N}_{2}$ ) even with the larger threshold value.
\end{abstract}

Key Words : Chemical kinetics reduction; Skeletal chemistry mechanism; Directed relation graph

碳氢物质是自然界最普遍的燃料之一, 碳氢氧 化的详细反应动力学机理一般包含数百或数千基元 反应, 涉及数十或数百种物质 ${ }^{[1-3]}$. 构筑碳氢燃料氧 化详细化学机理时, 需考虑温度、压力、燃油过量系 数、以及混合与驻留时间等参数能在宽广的范围内
变化, 因此, 碳氢详细机理一般较为复杂. 当前, 数值 模拟技术在燃烧装置的设计以及燃烧机理的研究中 起着越来越重要的作用, 但复杂的反应机理使得即 使是较为简单的燃烧现象也难于模拟, 如非稳态、多 维层流火焰. 复杂的化学机理除了使计算量大得难

Received: November 7, 2008; Revised: January 12, 2009; Published on Web: February 25, 2009.

*Corresponding author. Email: rqh@ustc.edu.cn.

国家自然科学基金(50676091, 50876097, 50536030)、教育部新世纪优秀人才支持计划(NCET-06-0546)、国家科技支撑计划(2006BAK06B04-2) 以及中国科学技术大学青年基金资助项目 
以承受外, 在计算理论上也带来了巨大难题, 各种化 学组分及其涉及的反应其特征时间尺度差异巨大, 详细反应使得研究和计算的对象变为极强的刚性系 统, 该系统的可靠和精密求解是较为困难的 ${ }^{[1-3]}$. 在 进行耦合燃料复杂化学机理的反应流模拟研究中我 们也发现 ${ }^{[4,5]}$, 涉及湍流和多维, 针对塊合燃料复杂化 学机理的多维反应流计算, 其痕迹组分的浓度值经 常是负值, 其主要原因就是上述的刚性问题. 采用美 国 Sandia 国家实验室发展的 CHEMKIN 软件包 ${ }^{[6-8]}$ 中的解刚性系统算法器 LSODE(或 VODE)往往也 不能解决问题, 特别值得一提的是, 目前国内关于耦 合燃料复杂化学机理的多维反应流计算渐热, 但从 其采用的时空步长来看, 模拟的可靠性令人怀疑. Law 和 Pitsch ${ }^{[1-3]}$ 等学者指出, 实际碳氢燃料的详细 反应动力学模型需要简化, 以满足燃烧数值模拟的 需要. 需要指出的是, 简化机理不能理解为简单的无 限快“总包”反应, 总包反应不能描述燃烧毒物形成 的“慢”反应过程, 不能研究熄火、重燃等火焰机理. 与详细机理相比, 简化机理具有较少的变量和较低 的刚性, 但仍能维持详细机理的全面性以及可靠的 预测精度.

复杂化学机理简化一直是国际燃烧学和反应动 力学界的主要研究方向之一, 对目前存在的机理简 化方法的描述可参见文献[9], 运用较多的机理简 化方法主要基于反应系统时间尺度分析基础上, 快 时间尺度的准稳态组分或偏平衡反应被去除 ${ }^{[10-16]}$. 本

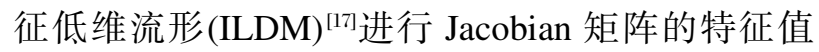
分析, 假定快的亚空间迅速消失, 在使用时常采用局 域自适应建表 ISAT 来降低计算量 ${ }^{[18,19]}$. 计算奇异摄 动 CSP 考虑 Jacobian 矩阵的时间相关性, 通过定义 快模式来获得简化机理 ${ }^{[20-24]}$. 基于时间尺度分析, 通 过去除较快特征时间尺度的组分和反应能够减少组 分数以及弱化系统刚性, 但求解简化机理相应的代 数方程组仍将消耗大量的 CPU 时间, 特别是针对较 为复杂的机理, 因此, 在进行基于时间尺度简化前, 往往需要查明和去除不重要的组分和基元反应, 即 获得框架化学机理. 目前有几种获得框架机理的方 法, 最早的方法基于敏感性分析 $\mathrm{SA}^{[25,26]}$, 该方法应用 较为简单, 但是它不能提供解耦信息, 同时后处理工 作量大且对研究者的经验有一定要求. 基于 SA 的 主成分分析 PCA, 通过敏感性矩阵的分析, 系统地 查明冗余反应 ${ }^{[27,28]}$. 直觉上, 反应简化也可以采用优 化的方法, 如遗传算法 $(\mathrm{GA})$, 但优化解强烈地依赖于
约束条件, 一般来说, 优化解收玫速度较慢. Wang 和 Frenklach ${ }^{[29]}$ 通过直接比较反应速率与预设临界 值, 提供了快速查明非重要反应的方法. 该方法的不 足在于没有考虑组分与反应的耦合, 因为在包含关 键自由基时, 较慢反应并不总是不重要的.

由上可以看出, 国际上对于由详细反应获得 框架化学机理已进行了大量研究, 但是包括直接 的组分去除方法尚未建立. 2005 年, Law等学者 ${ }^{[1]}$ 发 展了直接关系图(DRG)法, 2006和2008年, $\mathrm{Law}^{[2]}$ 和 $\mathrm{Pitsch}^{[3]}$ 等学者针对充分摚拌反应器(PSR)中碳氢燃 料着火等,进行了基于 DRG 的机理简化研究. 本文 的研究内容包括: 1) 研究基于 DRG 的机理简化程 序; 2) 提出通用的组分 $\mathrm{B}$ 对组分 $\mathrm{A}$ 生成的正规化贡 献公式, 可将 DRG 和其变形(基于 DRG 的误差传播 DRGEP 法)纳人其中, 同时可预见其它变形 DRG 方 法; 3) 将基于 DRG 的机理简化研究扩展到较 PSR 更为复杂的预混火焰的研究中, 确定有效范围, 获得 优化的适合预混火焰的甲烷分级简化动力学模型.

\section{1 方法与算法}

DRG 研究是 Law 等学者近期提出的一种重要 的复杂机理简化方法, 在我国尚未展开, 为较为清晰 地表述该思想以及论文的完整性，下列 DRG 方法、 扩展的 DRG 算法内容源自文献[1,2], DRGEP 内容 源自文献[3]. DRG 算法的程序实现以及变形 DRG 法表达为本文的工作.

\subsection{DRG 方法 ${ }^{[1,2]}$}

由于组分之间的复杂耦合, 查明和去除非重要 组分是困难的. 组分 $\mathrm{A}$ 与 $\mathrm{B}$ 之间可以通过共同出现 在快反应中而直接地强烈耦合, 也可以通过分别与 组分 $\mathrm{C}$ 的强烈作用而耦合, 从详细反应机理中去除 某单个组分一般需要去除一组与其强烈耦合的组 分. DRG 理论能很好地查明组分之间的复杂耦合关 系, DRG 中的节点代表详细机理中的每一组分, 如 果某组分 $\mathrm{B}$ 的去除将导致组分 $\mathrm{A}$ 的生成或消耗产 生较大误差, 即定义存在从 A 到 B 的边线. 图 1 示 出 $\mathrm{DRG}$ 中组分的典型关系 ${ }^{[1]}$, 假定开始节点 $\mathrm{A}$ 是机 理中的重要组分, 图 1(a)示出其它节点是否可达 $\mathrm{A}$. 图搜索法如 DFS(depth first search)能剥离复杂非线 性关系, 从而找到到达A的路径. 由图 1 (a), 框架化学 机理中应包含组分 $A 、 B$ 和 D. 注意到开始点集合中 并不需要包含机理中所有重要组分, 因为其它重要 组分可由 DFS 找出. Law 等 ${ }^{[1,2]}$ 认为惰性组分在可直 

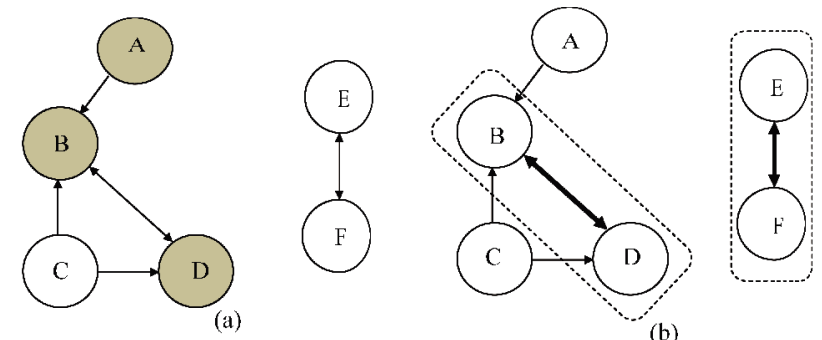

(b)

图 1 典型直接关系图 DRG 示例[1]

Fig.1 Schematics showing typical configuration of the directed relation graph (DRG) $)^{[1]}$

(a) basic configuration and identification of skeletal species; (b) demonstration of strongly coupled species groups

接加回到 DRG 框架化学机理中, 而我们在研究中 发现这样做是不行的, 会导致计算结果出现较大误 差. 很明显, DRG 框架化学机理中存在强烈耦合的 组分组, 图 1 (b) 示出强烈耦合的组分组 $[B, D]$ 和 $[E$, $F]$, 这样强烈耦合的组分组在机理中或者保持或者 整体去除.

组分 $\mathrm{A}$ 的生成或消耗速率为 $R_{\mathrm{A}}$, 表达为

$$
\begin{aligned}
& R_{\mathrm{A}}=\sum_{i=1, I} v_{\mathrm{A}, i} \omega_{i} \\
& \omega_{i}=k_{\mathrm{fi}} \prod_{j=1}^{K} C_{j}^{v_{j}^{\prime}}-k_{\mathrm{bi}} \prod_{j=1}^{K} C_{j}^{v_{j}^{\prime \prime}} \\
& k_{\mathrm{fi}}=\left[A_{i} T^{i i} \exp \left(-\frac{E_{i}}{R T}\right)\right] F_{i}
\end{aligned}
$$

式中, $i$ 和 $j$ 分别为第 $i$ 基元反应和第 $j$ 组分, $I$ 为基 元反应数, $K$ 为总组分数, $v_{\mathrm{A} i}$ 表示组分 $\mathrm{A}$ 的化学计 量系数(stoichiometric coefficients), $\omega_{i}$ 为生成速率, $k_{\mathrm{fi}}$ 和 $k_{\mathrm{b} i}$ 分别为向前和向后反应速率, $C_{j}$ 为摩尔浓 度, $v_{i j}^{\prime}$ 和 $v_{i j}^{\prime \prime}$ 分别为向前和向后计量系数, $A_{i}$ 为指前 因子, $T$ 为温度, $n i$ 表示第三个反应的温度指数, $E_{i}$ 表示活化能, $R$ 为通用气体常数, $F_{i}$ 为包含三体组分 的修正项. 定义组分 $\mathrm{B}$ 对组分 $\mathrm{A}$ 生成的正规化贡献 为

$$
r_{\mathrm{AB}}=\frac{\sum_{i=1, I}\left|v_{\mathrm{A}, i} \omega_{i} \delta_{\mathrm{B} i}\right|}{\sum_{i=1, I}\left|v_{\mathrm{A}_{i},} \omega_{i}\right|}
$$

其中,

$$
\delta_{\mathrm{B} i}= \begin{cases}1 & \text { (第 } i \text { 个基元反应包含 } \mathrm{B}) \\ 0 & \end{cases}
$$

可以看到, 正规化贡献 $r_{\mathrm{AB}}$ 足够大时, 去除组分 $\mathrm{B}$ 将 导致组分 $\mathrm{A}$ 的生成速率发生较大误差, 因此, 假如 $\mathrm{A}$ 保持, 则 $\mathrm{B}$ 也将保持, 称 $\mathrm{A}$ 强烈依赖 B. 执行中可
预设一个小的阈值 $\varepsilon$, 当 $r_{\mathrm{AB}}<\varepsilon$ 时, $\mathrm{A}$ 和 $\mathrm{B}$ 之间的依 赖可以忽略, 从 $\mathrm{A}$ 到 $\mathrm{B}$ 不存在边线.

基于 DRG 的去除非重要组分和基元反应的方 法与以前基于 Jacobian 分析 ${ }^{[9]}$ 的方法相比, 两类方 法都查明与机理中重要组分强烈耦合的组分. 首先, 由于没有 Jacobian 矩阵分析, DRG 法降低了简化计 算时间, 同时 DRG 能够根据给定精度, 查明所有备 选机理, 单次简化运行时间仅与机理图中边线数成 线性正比关系,而 Jacobian 分析一般要采用迭代算 法; 其次, 在 DRG 中可定义 0 和 1 之间的阈值, 从 而提供了简化机理的上下误差限; 第三, DRG 框架 化学机理包括了组分和基元反应的去除, 它可以直 接评价去除的作用, 而在 Jacobian 分析中, 是通过组 分浓度的局部摄动来评估.

\section{2 扩展的 DRG 算法 ${ }^{[2]}$}

DRG 简化有三个主要步骤, 即图型构筑、定义 框架化学机理组分的图型搜索步、以及创建最后机 理文件步. 在图型构筑步, 根据方程(4), 评价每一对 组分的相对误差, $K$ 个组分则共有 $K^{2}$ 项评价, 对于 $I$ 个基元反应, 每项包含 $I$ 次乘运算, 因此, 图型构筑 步中的平滑算法需要 $K^{2} I$ 次评价. 由于详细机理的 计量系数矩阵的稀疏性, 因此仅评估每一反应关于 关系边线的贡献可以大大提高运算效率. 在图型搜 索步, 图型中每一边与预设阈值 $\varepsilon$ 相比较, 采用 DFS 搜索可以获得框架机理的组分集, 该步的运算 量也是 $I$, 采用修正的 DFS 方法 RDFS 可以进一步 提高效率. 扩展的 DRG 算法如下:

步骤一, 图型构筑

反应循环 1 开始, $i=1-I$

循环 2 开始, 包含在反应 $i$ 中的组分对 $\mathrm{A}$ 和 $\mathrm{B}$ 估计 $v_{\mathrm{A} i} \omega_{i}$ 和 $\delta_{\mathrm{B} i}$

假如 $\mathrm{A} \rightarrow \mathrm{B}$ 没有初始化

重设关于 $\mathrm{A} \rightarrow \mathrm{B}$ 边的 $r_{\mathrm{AB}}$ 分子和分母 假设结束

循环 2 结束

循环 1 结束

循环 3 开始, 关于初始化边线

累积 $r_{\mathrm{AB}}$ 分子和分母项

循环 3 结束

步骤二, 图型搜索

调用模块 RDFS( )

步骤三, 创建最后机理

去除小于阈值 $\varepsilon$ 的组分 
去除相应反应

重写剩余组分和相关反应

结束

模块 RDFS( )

设机理中组分为结点, 从选中组分开始, 假设为 $\mathrm{A}$, 根据组分之间关系值 $r_{\mathrm{AB}}$ 的大小, 找出并标记最 大关系值的组分 $\mathrm{B}$, 以此类推.

\subsection{DRGEP ${ }^{[3]}$ 与变形 DRG 法}

近期, Pitsch 等学者 ${ }^{[3]}$ 提出基于 DRG 的误差传 播机理简化方法(DRGEP). DRGEP在计算 $r_{\mathrm{AB}}$ 时采用

$$
r_{\mathrm{AB}}=\frac{\left|\sum_{i=1, I} v_{\mathrm{A},} \omega_{i} \delta_{\mathrm{B} i}\right|}{\max \left(P_{\mathrm{A}}, C_{\mathrm{A}}\right)}
$$

其中,

$$
\begin{aligned}
P_{\mathrm{A}} & =\sum_{i=1, I} \max \left(0, v_{\mathrm{A},}\left(\omega_{i}\right)\right. \\
C_{\mathrm{A}} & =\sum_{i=1, I} \max \left(0,-v_{\mathrm{A},}\left(\omega_{i}\right)\right.
\end{aligned}
$$

方程(6)的分子可推导为

$$
\begin{aligned}
\left|\sum_{i=1, I} v_{\mathrm{A}, i} \omega \delta_{\mathrm{B} i}\right| & =\mid \sum_{i=1, I} \max \left(0, v_{\mathrm{A},}, \omega_{i}\right) \delta_{\mathrm{B} i}{ }^{-} \\
\sum_{i=1, I} \max \left(0,-v_{\mathrm{A},} \omega \delta_{\mathrm{B} i}\right) \mid & =\left|P_{\mathrm{AB}}-C_{\mathrm{AB}}\right|
\end{aligned}
$$

上式右边对应组分 $\mathrm{A}$ 在包含组分 $\mathrm{B}$ 的反应中的生 成 $\left(P_{\mathrm{AB}}\right)$ 和消耗 $\left(C_{\mathrm{AB}}\right)$, 由于 $0 \leqslant P_{\mathrm{AB}} \leqslant P_{\mathrm{A}}$ 和 $0 \leqslant C_{\mathrm{AB}} \leqslant$ $C_{\mathrm{A}}$, 因此 $-C_{\mathrm{A}} \leqslant P_{\mathrm{AB}}-C_{\mathrm{AB}} \leqslant P_{\mathrm{A}}$, 它等价于 $\left|P_{\mathrm{AB}}-C_{\mathrm{AB}}\right| \leqslant$ $\max \left(P_{\mathrm{A}}, C_{\mathrm{A}}\right)$, 该不等式说明, 组分 $\mathrm{B}$ 对于组分 $\mathrm{A}$ 的净 贡献不能超过组分 $\mathrm{A}$ 的总生成和消耗.

注意到如果方程(4)改写为

$$
r_{\mathrm{AB}}=\frac{\sum_{i=1, I}\left|v_{\mathrm{A}, i} \omega_{i} \delta_{\mathrm{B} i}\right|}{\sum_{i=1, I}\left|v_{\mathrm{A}, i} \omega_{i}\right|}=\frac{|| \sum_{i=1, I}\left(v_{\mathrm{A},} \omega_{i} \delta_{\mathrm{Bi}}\right)||}{\|\left|\sum_{i=1, I}\left(v_{\mathrm{A},} \omega_{i}\right)\right| \mid}
$$

式中分子分母为范数形式, 我们称为变形的 DRG 法. 很明显, DRG 和 DRGEP 可以统一到范数形式 下, 范数的具体表达方式可以有多种. 因此, 可以推 断, 一定存在其它变形的 DRG 法, 如最小二乘形式 等, 可以预见, 不同的变形 DRG 法将适合不同火焰 结构形式的简化.

\section{2 方法展示: 甲烷框架化学机理}

\section{1 低碳烷烃详细化学反应动力学}

选择 Konnov 的低碳烷烃 0.5 版氧化机理 ${ }^{[30]}$ 作
为需要简化的详细反应动力学模型, Konnov 0.5 版 机理包含 1207 个基元反应、涉及 127 种物质, 该机 理的有效性可参见文献[30].

\section{2 甲烷 DRG 框架化学机理构筑}

选择甲烷自由扩散预混火焰作为物理对象, 火 焰结构参数为: 压力 $1 \times 10^{5} \mathrm{~Pa}$, 流量 $0.04 \mathrm{~g} \cdot \mathrm{cm}^{-2} \cdot \mathrm{s}^{-1}$, 初始温度 $298 \mathrm{~K}$. 选取 CHEMKIN-II ${ }^{[6-8]}$ 源码作为火 焰计算工具, 考虑多组分扩散和热扩散, 自适应网格 参数 $\mathrm{GRAD}=0.1 、 \mathrm{CURV}=0.5$, 相对和绝对误差分别 为 $1.0 \times 10^{-4}$ 和 $1.0 \times 10^{-9}$.

为了使获得的甲烷 DRG 框架化学机理在宽广 的参数范围内有效, 选取的采样空间范围是: 燃油 过量系数为 0.7 (稀薄燃烧) -1.3 (富油燃烧), 温度为 300-2000 K. 选取甲烷作为开始组分. 图 2 示出框架 机理中组分数、相应基元反应数与给定阈值 $\varepsilon$ 之间 的关系, 可以发现: 1) $\varepsilon$ 趋向零, 框架化学机理的组 分数与反应数趋向详细化学机理的组分数与反应 数, 即框架化学机理接近详细机理; 当 $\varepsilon$ 接近 1 , 框 架机理中仅能维持初始选取的组分, 其它的耦合都 将截断; 2) 在某些 $\varepsilon$ 值, 框架机理中组分和反应数 会发生跳跃现象, 组分和反应数并不是 $\varepsilon$ 的连续函 数, 这是由于存在不同强度耦合的组分组, 这些组分 组或者被保留在机理中或者被去除.

研究中发现, 当仅选取甲烷作为开始组分, 在 $\varepsilon$ 不接近于零时, 形成的 DRG 机理中不包含 $\mathrm{N}_{2}$. 由于 机理在实际使用时一般给定初始的 $\mathrm{CH}_{4}$ 和空气 $\left(\mathrm{O}_{2}+\right.$ $\mathrm{N}_{2}$ ) 量, 因此当我们在形成的DRG机理组分中加人 $\mathrm{N}_{2}$, 采用简化机理和详细机理的预混火焰计算结果 差异巨大, 所以, 需要同时选取 $\mathrm{CH}_{4}$ 和 $\mathrm{O}_{2}$ 作为开始

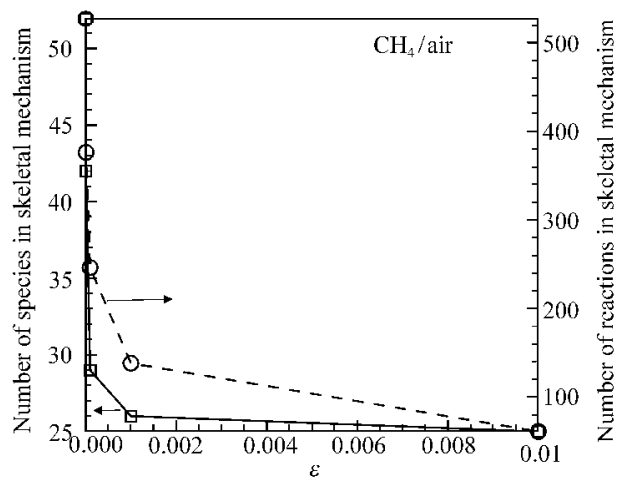

图 2 框架机理中组分数、相应基元反应数与给定阈值 $\boldsymbol{\varepsilon}$ 之间的关系

Fig.2 Dependence of the species and reaction number of the skeletal mechanism on the threshold value $\varepsilon$ 
组分. 当给定的 $\varepsilon$ 为 0.001 时, 获得构筑框架化学机 理含 339 个反应、涉及 42 种组分, 42 种组分是: 1) $\mathrm{H}$, 2) $\mathrm{H}_{2}$, 3) $\mathrm{O}$, 4) $\mathrm{O}_{2}$, 5) $\mathrm{OH}$, 6) $\mathrm{HO}_{2}$, 7) $\mathrm{H}_{2} \mathrm{O}$, 8) $\mathrm{H}_{2} \mathrm{O}_{2}$, 9) $\left.\left.\left.\left.\mathrm{CO}, 10) \mathrm{CO}_{2}, 11\right) \mathrm{HCO}, 12\right) \mathrm{CH}_{3}, 13\right) \mathrm{CH}_{4}, 14\right) \mathrm{C}_{2} \mathrm{H}_{6}$, 15) $\mathrm{CH}_{2} \mathrm{O}$, 16) $\mathrm{C}_{2} \mathrm{H}_{5}$, 17) $\mathrm{CH}_{2}$, 18) $\mathrm{CH}_{3} \mathrm{O}$, 19) $\mathrm{CH}_{2} \mathrm{OH}$, 20) $\left.\mathrm{CH}, 21) \mathrm{C}_{2} \mathrm{H}_{2}, 22\right) \mathrm{C}_{2} \mathrm{H}_{4}$, 23) $\mathrm{C}_{2} \mathrm{H}_{3}$, 24) $\left.\mathrm{CH}_{3} \mathrm{OH}, 25\right)$ $\left.\left.\left.\left.\mathrm{CH}_{3} \mathrm{HCO}, 26\right) \mathrm{HCCO}, 27\right) \mathrm{SCH}_{2}, 28\right) \mathrm{CH}_{3} \mathrm{CO}, 29\right)$ $\left.\left.\left.\mathrm{CH}_{3} \mathrm{CO}_{3}, 30\right) \mathrm{CH}_{3} \mathrm{O}_{2}, 31\right) \mathrm{CH}_{3} \mathrm{O}_{2} \mathrm{H}, 32\right) \mathrm{C}_{2} \mathrm{H}_{5} \mathrm{O}_{2} \mathrm{H}, 33$ ) $\left.\left.\mathrm{C}_{2} \mathrm{H}_{5} \mathrm{O}_{2}, 34\right) \mathrm{C}_{2} \mathrm{H}_{5} \mathrm{OH}, 35\right) \quad \mathrm{SC}_{2} \mathrm{H}_{5} \mathrm{O}$, 36) $\mathrm{PC}_{2} \mathrm{H}_{5} \mathrm{O}, 37$ ) $\mathrm{CH}_{2} \mathrm{HCO}$, 38) $\left.\left.\left.\mathrm{C}_{3} \mathrm{H}_{6}, 39\right) \mathrm{IC}_{3} \mathrm{H}_{7}, 40\right) \mathrm{C}_{3} \mathrm{H}_{3}, 41\right) \mathrm{Ar}, 42$ ) $\mathrm{N}_{2}$; 如给定的 $\varepsilon$ 为 0.01 , 最后获得构筑框架化学机理 含 182 个反应、涉及 27 种组分, 27 种组分是: 1) H, 2) $\left.\mathrm{H}_{2}, 3\right) \mathrm{O}$, 4) $\mathrm{O}_{2}$, 5) $\left.\left.\mathrm{OH}, 6\right) \mathrm{HO}_{2}, 7\right) \mathrm{H}_{2} \mathrm{O}$, 8) $\mathrm{H}_{2} \mathrm{O}_{2}$, 9) $\left.\left.\left.\left.\mathrm{CO}, 10) \mathrm{CO}_{2}, 11\right) \mathrm{HCO}, 12\right) \mathrm{CH}_{3}, 13\right) \mathrm{CH}_{4}, 14\right) \mathrm{CH}_{2} \mathrm{O}$, 15) $\mathrm{C}_{2} \mathrm{H}_{5}$, 16) $\mathrm{CH}_{2}$, 17) $\mathrm{CH}_{3} \mathrm{O}$, 18) $\mathrm{CH}_{2} \mathrm{OH}$, 19) $\mathrm{C}_{2} \mathrm{H}_{4}$, 20) $\left.\left.\left.\mathrm{C}_{2} \mathrm{H}_{3}, 21\right) \mathrm{CH}_{3} \mathrm{OH}, 22\right) \mathrm{SCH}_{2}, 23\right) \mathrm{CH}_{3} \mathrm{O}_{2}, 24$ ) $\left.\mathrm{CH}_{3} \mathrm{O}_{2} \mathrm{H}, 25\right) \mathrm{N}_{2}$, 26) $\left.\mathrm{Ar}, 27\right) \mathrm{C}_{2} \mathrm{H}_{6}$. 二个框架机理见 supporting informaion (available freely from www. whxb.pku.edu.cn).

\section{3 有效性}

上述将包含 1207 个基元反应、涉及 127 种物质 的 Konnov 反应动力学模型简化为二个 DRG 框架 化学机理: 339 机理和 182 机理. 为展示所获 DRG 机理的精度和计算可靠性, 将耦合 DRG 182 机理和 其详细机理的预混火焰模拟结果进行对比. 本研究 的燃油过量系数 $\varphi$ 定义为 $\varphi=\left(n_{\text {air }} / n_{\mathrm{f}}\right)_{\mathrm{s}} \mathrm{l} /\left(n_{\mathrm{air}} / n_{\mathrm{f}}\right)_{\text {act }}$, 其中, $\left(n_{\mathrm{ail}} / n_{\mathrm{f}}\right)_{\mathrm{st}}$ 表示等化学计量比时的空气和燃油摩尔数 之比, $\left(n_{\text {ail }} / n_{\mathrm{f}}\right)_{\text {act }}$ 表示实际空气和燃油摩尔数之比.

图 3 表示在不同燃油过量系数下, 框架机理与

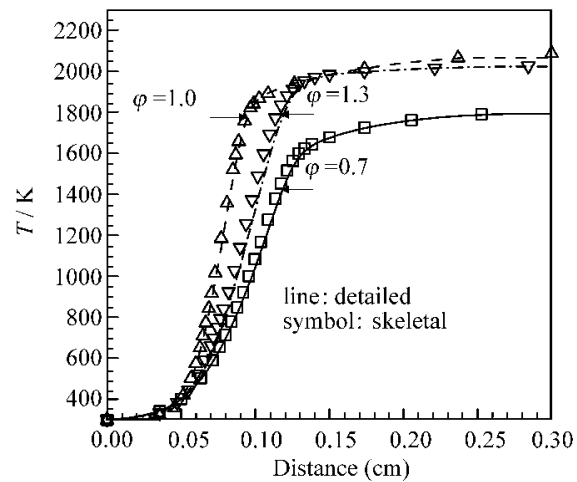

图 3 预混火焰中不同燃油过量系数时框架机理与 详细机理的温度随火焰高度变化

Fig.3 Dependence of temperature on the distance between the detailed and skeletal mechanisms at various air-fuel equivalence ratios in premixed flame

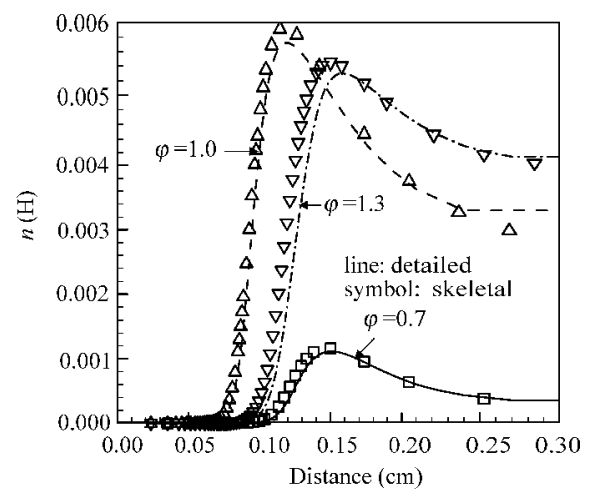

图 4 预混火焰中不同燃油过量系数时框架机理与详细机 理的 $\mathbf{H}$ 摩尔分数随火焰高度变化

Fig.4 Dependence of molar fraction radical $\mathrm{H}$ on the distance between the detailed and skeletal mechanisms at various air-fuel equivalence ratios in premixed flame

详细机理的温度随火焰高度变化的预测值, 可以发 现, 稀薄燃烧温度较低, 富油燃烧温度较高, 但与等 燃油过量系数火焰相比温度上升较慢. 图 4 和图 5 分别表示火焰中重要自由基物质 $\mathrm{H}$ 和 $\mathrm{OH}$ 的摩尔 分数随火焰高度的变化, $\mathrm{H}$ 和 $\mathrm{OH}$ 是维持燃烧过程 的重要活性物质, 也是燃烧场诊断的重要标志性物 质, 它们生成与消耗主要在“自由基池”中进行: $\mathrm{H}+$ $\mathrm{O}_{2}=\mathrm{OH}+\mathrm{O}, 2 \mathrm{OH}=\mathrm{H}_{2} \mathrm{O}+\mathrm{O}, \mathrm{O}+\mathrm{H}_{2}=\mathrm{OH}+\mathrm{H}, \mathrm{H}_{2}+\mathrm{OH}=$ $\mathrm{H}_{2} \mathrm{O}+\mathrm{H}, \mathrm{H}+\mathrm{OH}+\mathrm{M}=\mathrm{H}_{2} \mathrm{O}+\mathrm{M} \quad(\mathrm{M}$ 表示三体物质 (third-body)), $2 \mathrm{H}+\mathrm{M}=\mathrm{H}_{2}+\mathrm{M}$. 值得注意的是, 稀薄燃 烧的 $\mathrm{OH}$ 比富油燃烧时的摩尔分数较高, 稀薄燃烧 和富油燃烧时的 $\mathrm{OH}$ 摩尔分数都比等燃油过量系数 时低得多;而稀薄燃烧的 $\mathrm{H}$ 比富油燃烧时的摩尔分 数较低, 同时, 富油燃烧时的 $\mathrm{H}$ 摩尔分数和等燃油

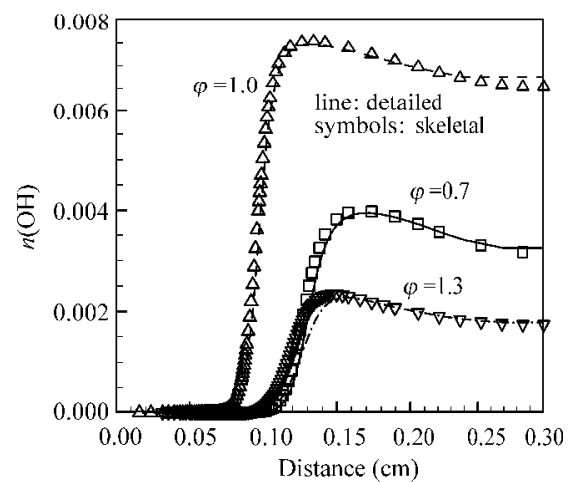

图 5 预混火焰中不同燃油过量系数时框架机理与详细机 理的 $\mathrm{OH}$ 摩尔分数随火焰高度变化

Fig.5 Dependence of molar fraction radical $\mathrm{OH}$ on the distance between the detailed and skeletal mechanisms at various air-fuel equivalence ratios in premixed flame 


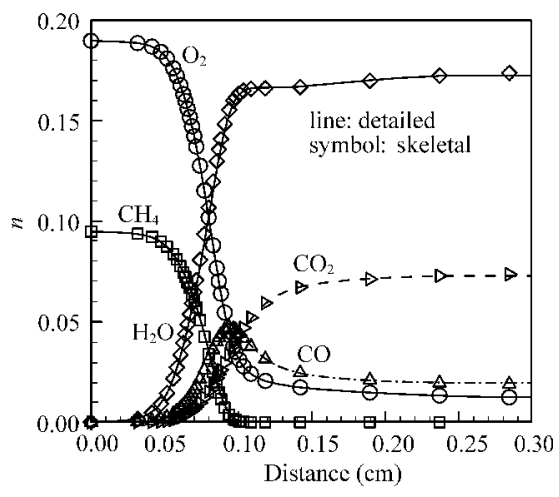

图 6 预混火焰中等燃油过量系数时框架机理与 详细机理的主要物质摩尔分数随火焰高度变化

Fig.6 Dependences of molar fraction main species on the distance between the detailed and skeletal mechanisms at air-fuel equivalence ratio $\varphi=1$ in premixed flame

过量系数时相当但生成滞后. 图 6 为等燃油过量系 数下, 框架机理与详细机理的主要物质摩尔分数随 火焰高度变化的预测值, 明显地, 燃料 $\mathrm{CH}_{4}$ 和氧化 剂 $\mathrm{O}_{2}$ 不断消耗, 主产物 $\mathrm{CO}_{2}$ 和 $\mathrm{H}_{2} \mathrm{O}$ 不断生成, 而主产 物 $\mathrm{CO}$ 有一个生成和消耗的过程, 这是由于 $\mathrm{CO}_{2}$ 和 $\mathrm{H}_{2} \mathrm{O}$ 比CO稳定.

由图 3 到图 6 可以看出, 框架机理对于火焰结 构的预测结果与详细机理计算结果吻合很好, 表明 获得的 DRG 机理有较高的可靠性. 目前, 国际上关 于甲烷燃烧的著名机理为 GRI-Mech2.11 和 GRIMech $3.0^{[31]}$, 分别为 277 个反应涉及 49 种组分、 325 个 反应涉及 53 种组分. 所获得二个 DRG 机理与 GRIMech 机理规模相当, 当然, 阈值 $\varepsilon$ 越大, DRG 机理 越简化. DRG 框架机理大大缩短了计算时间, 以使 用上述 DRG 182 机理为例, 等燃油过量系数的预混 火焰计算仅需 CPU 时间 $22 \mathrm{~s}$, 而耦合 Konnov 详细 机理的计算需 CPU 时间 $754 \mathrm{~s}$.

\section{3 结论与展望}

采用直接关系图 DRG 法, 针对碳氢类氧化反 应详细机理, 成功地进行了不同指定精度水平的框 架化学机理简化研究, 并发展出相应程序. 通过 DRG 可以发现, 机理中存在强烈耦合的组分组, 在 框架机理中, 这些组分组或同时保持或集体去除. 研 究表明, 给定阈值 $\varepsilon$ 为 0.01 的框架机理仅包含 182 个反应、涉及 27 种组分, 虽然给定阈值的精度并不 高, 但所得框架机理与原详细机理关于火焰结构的 计算结果吻合很好. 研究发现, 开始组分仅给定燃料
物质是不够的, 这与 Law 和 Pitsch 等不一致, 当开 始组分仅给定燃料物质, 则阈值 $\varepsilon$ 需很小, 形成的框 架机理较大, 否则计算误差不可接受, 而开始组分给 定燃料和氧化剂(或 $\mathrm{N}_{2}$ )物质, 阈值 $\varepsilon$ 可以取得较大, 形成的框架机理尺度可以较小, 而火焰模拟的精度 仍很高. DRG 法的另一个很重要的作用是可以查明 与燃烧中某组分关联密切的组分和反应, 如, 当我们 关注碳黑形成时, 可以选取多环芳香烃 PAH 作为指 定的开始物质, 从而获得不同水平的碳黑形成亚 机理.

DRG 是近期国际上提出的一种复杂机理简化 方法, 我们提出 DRG 的统一计算公式, 可将 DRG 和其变形 DRGEP 统一起来, 可以预见, 其中所取范 数形式不同, 可获得不同的 DRG 以适应不同的火 焰结构计算和机理简化, 特别地, 范数中不仅包含反 应速率, 还可考虑包含流动参数.

Supporting Information Available: The two skeletal mechanisms for methane have been included. This information is available free of charge via the internet at http://www.whxb.pku.edu.cn.

\section{References}

1 Lu, T. F.; Law, C. K. A directed relation graph method for mechanism reduction. In: 30th symposium (international) on combustion. Pittsburgh: The Combustion Institute, 2005: 13331341

2 Lu, T. F.; Law, C. K. Combust. Flame, 2006, 144(1-2): 24

3 Pepiot-Desjardins, P.; Pitsch, H. Combust. Flame, 2008, 154(1-2): 67

4 Jiang, Y.; Qiu, R.; Fan, W. C. Progress in Natural Science, 2002, 12(4): 442 [蒋 勇, 邱 榕, 范维澄. 自然科学进展, 2002, 12 (4): 442]

5 Jiang, Y.; Qiu, R.; Dong, G.; Fan, W. C. Journal of Combustion Science and Tecnology, 2005, 11(2): 109 [蒋 勇, 邱 榕, 董＼cjkstart钢, 范维澄. 燃烧科学与技术, 2005,11(2): 109]

6 Kee, R. J.; Rupley, F. M.; Miller, J. A. Chemkin-II: a Fortran chemical kinetics package for the analysis of gas-phase chemical kinetics. Report SAND89-8009. Sandia, 1989

7 Kee, R. J.; Grcar, J. F.; Smooke, M. D.; Miller, J. A. PREMIX: a Fortran program for modeling steady laminar one-dimensional premixed flames. Report SAND85-8240. Sandia, 1985

8 Lutz, A. E.; Kee, R. J.; Miller, J. A. SENKIN: a Fortran program for predicting homogeneous gas phase chemical kinetics with sensitivity analysis. Report SAND87-8248. Sandia, 1987

9 Valorani, M.; Creta, F.; Goussis, D. A.; Lee, J. C. Combust. Flame, 2006, 146(1-2): 29

10 Peters, N.; Williams, F. A. Combust. Flame, 1987, 68(2): 185 
11 Seshadri, K.; Peters, N. Combust. Flame, 1988, 73(1): 23

12 Turányi, T.; Tomlin, A. S.; Pilling, M. J. J. Phys. Chem., 1993, 97 (1): 163

13 Sung, C. J.; Law, C. K.; Chen, J. Y. An augmented reduced mechanism for methane oxidation with comprehensive global parametric validation. In: 27th symposium (international) on combustion. Pittsburgh: The Combustion Institute, 1998: 295-304

14 Sung, C. J.; Law, C. K.; Chen, J. Y. Combust. Flame, 2001, 125 (1-2): 906

15 Turányi, T.; Tóth, J. Acta Chimica Hungarica, 1992, 129(6): 903

16 Soyhan, H. S.; Mauss, F.; Sorusbay, C. Combust. Sci. Tech., 2002, 174(11-12): 73

17 Maas, U.; Pope, S. B. Combust. Flame, 1992, 88(3-4): 239

18 Pope, S. B. Combust. Theor. Model., 1997, 1(1): 41

19 Yang, B.; Pope, S. B. Combust. Flame, 1998, 112(1): 85

20 Lam, S. H.; Goussis, D. A. Understanding complex chemical kinetics with computational singular perturbation. In: 22th symposium (international) on combustion. Pittsburgh: The Combustion Institute, 1988: 931-941

21 Lam, S. H.; Goussis, D. A. Int. J. Chem. Kinet., 1994, 26: 461
22 Massias, A.; Diamantis, D.; Mastorakos, E.; Mastorakos, D. A. Combust. Flame, 1999, 117(4): 685

23 Lu, T. F.; Ju, Y.; Law, C. K. Combust. Flame, 2001, 126(1): 1445

24 Valorani, M.; Najm, H. N.; Goussis, D. A. Combust. Flame, 2003, 134(1-2): 35

25 Rabitz, H.; Kramer, M.; Dacol, D. Annu. Rev. Phys. Chem., 1983, 34: 419

26 Turányi T. J. Math. Chem., 1990, 5(3): 203

27 Vajada, S.; Valko, P.; Turányi, T. Int. J. Chem. Kinet., 1985, 17: 55

28 Dong, G.; Qiu, R.; Jiang, Y.; Zhang, H. P. Fire Safety Science, 2004, 13(3): 158 [董 刚, 邱 榕, 蒋 勇, 张和平. 火灾科学, 2004, 13(3): 158]

29 Wang, H.; Frenklach, M. Combust. Flame, 1991, 87(3-4): 365

30 Konnov, A. Detailed reaction mechanism for small hydrocarbons combustion. Release 0.5. http://homepages. vub.ac.be/ akonnov/, 2000

31 Smith, G. P.; Golden, D. M.; Frenklach, M.; Moriarty, N. W.; Eiteneer, B.; Goldenberg, M.; Bowman, C. T.; Hanson, R. K.; Song, S.; Gardiner, Jr. W. C. ; Lissianski, V. V.; Qin, Z. W. http:// www.me.berkeley.edu/gri_mech/ 